\title{
Free-Form Optimization of Thin-Walled Structure for Frequency Response Problem
}

\author{
Masatoshi Shimoda ${ }^{1}$ and Yang Liu $^{2}$ \\ ${ }^{1}$ Department of Advanced Science and Technology, Toyota Technological Institute, 2-12-1 Hisakata, Tenpaku-ku, \\ Nagoya 468-8511, Japan \\ ${ }^{2}$ Department of Mechanical Engineering, Sojo University, 4-22-1 Ikeda, Nishi-ku, Kumamoto 860-0082, Japan
}

Correspondence should be addressed to Yang Liu; liuyang60212@gmail.com

Received 24 February 2015; Accepted 3 August 2015

Academic Editor: Emiliano Mucchi

Copyright (c) 2015 M. Shimoda and Y. Liu. This is an open access article distributed under the Creative Commons Attribution License, which permits unrestricted use, distribution, and reproduction in any medium, provided the original work is properly cited.

\begin{abstract}
We present a node-based free-form optimization method for designing forms of thin-walled structures in order to control vibration displacements or mode at a prescribed frequency. A squared displacement error norm is introduced at the prescribed surface as the objective functional to control the vibration displacements to target values in a frequency response problem. It is assumed that the thin-walled structure is varied in the normal direction to the surface and the thickness is constant. A nonparametric shape optimization problem is formulated, and the shape gradient function is theoretically derived using the material derivative method and the adjoint variable method. The shape gradient function obtained is applied to the surface of the thin-walled structure as a fictitious traction force to vary the form. With this free-form optimization method, an optimum thin-walled structure with a smooth free-form surface can be obtained without any shape parameterization. The calculated results show the effectiveness of the proposed method for the optimal free-form design of thin-walled structures with vibration mode control.
\end{abstract}

\section{Introduction}

Vibrations and attendant noise in architectural and civil structures, automobiles, aircraft, electric appliances, and other structures not only cause discomfort to users, but also give rise to strength problems such as fatigue life issues. Various measures have been implemented over the years to isolate vibration sources and transfer paths, to reduce vibration levels, and to control vibration modes. On the other hand, thin-walled structures such as curved or flat panels constitute one of the principal structural elements of these structures mentioned above. Because of their thinness and lightness, thin-walled structures are apt to become the sources of vibration. These structural elements always require countermeasures against vibration and noise in the structural design process. The purpose of this study is to develop a method for finding the optimal form of a thin-walled structure in order to control vibration displacements or a vibration mode to prescribed target values in frequency response problems of vibrating thin-walled structures.
Hanging models are often used as techniques for experimentally finding the optimal forms of thin-walled structures or shells. While such methods are convenient to use, they can only be applied under certain limited boundary conditions. Meanwhile, progress in research on computer-based numerical shape optimization methods has led to proposals for a variety of shape optimization techniques. Computerbased numerical shape optimization methods can be broadly divided into two categories that are used selectively according to the intended objective. One category comprises parametric methods whereby the design variables for determining the shape are parameterized in advance and a mathematical programming method is applied to determine the values of the parameters. Examples of the proposed techniques of this type include the basis vector method and methods that use parametric surfaces such as Bezier surfaces [1-3]. Because these methods use few design variables, they are compatible with CAD systems and allow high computational efficiency, but one drawback is that the shape obtained is strongly dependent on the parameters. Since the late 1980s, 


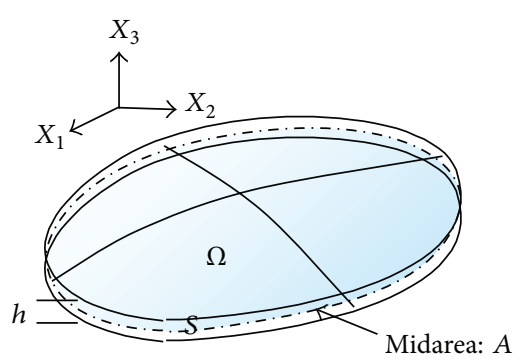

(a) Geometry of a thin-walled structure

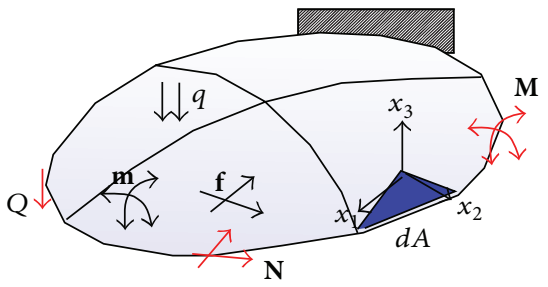

(b) External forces

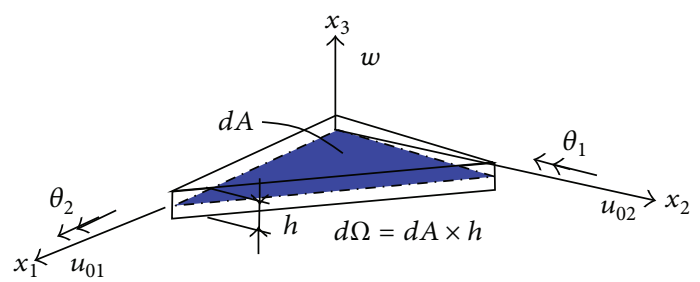

(c) D.O.F. and sign convention

FIGURE 1: Geometry of a thin-walled structure consisting of infinitesimal flat surfaces.

parametric methods have been the central focus of research and many reports have been presented concerning design variable parameterization [4].

The other category comprises parameter-free methods that treat all nodes as design variables without requiring any design variable parameterization. The drawbacks of parameter-free methods have included the problems of largescale design variables and the occurrence of jagged shape or the zigzag surface problem [5]. The former problem is often resolved by using the adjoint variable method in the sensitivity analysis, and the use of a filtering technique has been proposed as a solution to the jagged surface problem that is a drawback of node-by-node procedures [6-9]. Proposals for parameter-free methods are vastly fewer in number than their parametric counterparts. The authors have previously proposed a free-form optimization method [10]. This method is based on the traction method [11], which is a type of gradient method in a Hilbert space. The use of force for varying the surface reduces the objective functional while maintaining surface smoothness. We have so far applied this method to stiffness problems [10] and shape identification problem [12] and have also demonstrated its applicability to the structural design of automotive parts [13].

This paper describes the application of our proposed method to a problem of controlling the vibration displacements of a thin-walled structure in an effort to obtain the optimal free-form shape under mode control. A distributedparameter shape identification problem was formulated in which the objective functional was defined as the squared error of the target amplitude relative to a prescribed frequency in a prescribed domain. The sensitivity function (i.e., shape gradient function) of the objective functional with respect to shape variation in the out-of-plane direction to the surface was derived and applied to the proposed free-form optimization method. The validity of the proposed method was verified by applying it to several typical design problems. The following sections first describe the governing equation of thin-walled structures and next the formulation of the freeform optimization problem, the proposed method, and then the calculated results obtained.

\section{Governing Equation of Thin-Walled Structure Assembled by Infinitesimal Flats}

As shown in Figure 1(a) and (1), consider a thin-walled structure having an initial bounded domain $\Omega \subset \mathbb{R}^{3}$ (boundary of $\partial \Omega$ ), midarea $A$ (boundary of $\partial A$ ), side surface $S$, and plate thickness $h$. It is assumed for simplicity that stress and strain of the thin-walled structure are expressed by superposing the membrane and bending components and by neglecting their coupling effect. The Mindlin-Reissner plate theory is applied concerning plate bending. Consider

$$
\begin{aligned}
\Omega & =\left\{\left(x_{1}, x_{2}, x_{3}\right) \in \mathbb{R}^{3} \mid\left(x_{1}, x_{2}\right) \in A \subset \mathbb{R}^{2}, x_{3}\right. \\
& \left.\in\left(-\frac{h}{2}, \frac{h}{2}\right)\right\}, \\
\Omega & =A \times\left(-\frac{h}{2}, \frac{h}{2}\right), \\
S & =\partial A \times\left(-\frac{h}{2}, \frac{h}{2}\right) .
\end{aligned}
$$

In addition, it is assumed that the mapping of the local coordinate system $\left(x_{1}, x_{2}, 0\right)$ which gives the position of the midarea of the plate to the global coordinate system $\left(X_{1}, X_{2}, X_{3}\right)$, that is, $\Phi:\left(x_{1}, x_{2}, 0\right) \in \mathbb{R}^{3} \mapsto\left(X_{1}, X_{2}, X_{3}\right) \in$ $\mathbb{R}^{3}$, is piecewise smooth. Planar triangular shell elements are used to discretize thin-walled structures in this work. 
Using the sign convention in Figure 1(c), the displacement expressed by the local coordinates $\mathbf{u}=\left\{u_{i}\right\}_{i=1,2,3}$ is considered by dividing it into the displacement in the in-plane direction $\left\{u_{\alpha}\right\}_{\alpha=1,2}$ and the displacement in the out-of-plane direction $u_{3}$. Considering a plane stress condition as shown in (2), the displacements $u_{\alpha}$ and $u_{3}$ can be expressed as (3) and (4), respectively, by using the Mindlin-Reissner plate theory [14]. Hence,

$$
\begin{aligned}
\sigma_{33} & =0, \\
u_{\alpha}\left(x_{1}, x_{2}, x_{3}\right) & \equiv u_{0 \alpha}\left(x_{1}, x_{2}\right)-x_{3} \theta_{\alpha}\left(x_{1}, x_{2}\right), \\
u_{3}\left(x_{1}, x_{2}, x_{3}\right) & \equiv w\left(x_{1}, x_{2}\right),
\end{aligned}
$$

where $\left\{u_{0 \alpha}\right\}_{\alpha=1,2}, w$, and $\left\{\theta_{\alpha}\right\}_{\alpha=1,2}$ indicate the in-plane displacement, out-of-plane displacement, and rotational angle of the midarea of the plate, respectively. Then, the weak form equation of the steady-state forced vibration relative to $\left(\mathbf{u}_{0}, w, \boldsymbol{\theta}\right) \in U$ can be expressed as (5) by substituting (2)-(4) into the variational equation (i.e., weak form) of the threedimensional linear elastic theory, eliminating $\varepsilon_{33}$. As shown in Figure 1(b), forces acting relative to the local coordinate system $\left(x_{1}, x_{2}\right)$ on the domain $A$ and the boundary $\partial A_{g}(\mathrm{C}$ $\partial A$ ) are defined as follows: an out-of-plane load $q$ per unit area, an in-plane load $\mathbf{f}=\left\{f_{\alpha}\right\}_{\alpha=1,2}$ and an out-of-plane moment $\mathbf{m}=\left\{m_{\alpha}\right\}_{\alpha=1,2}$ per unit area, an in-plane load $\mathbf{N}=$ $\left\{N_{\alpha}\right\}_{\alpha=1,2}$ per unit length, a shearing force $\mathbf{Q}$ per unit length, and a bending moment $\mathbf{M}=\left\{M_{\alpha}\right\}_{\alpha=1,2}$ per unit length. Consider the following:

$$
\begin{gathered}
a\left(\left(\mathbf{u}_{0}, w, \boldsymbol{\theta}\right),\left(\overline{\mathbf{u}}_{0}, \bar{w}, \overline{\boldsymbol{\theta}}\right)\right)-\omega^{2} b\left(\left(\mathbf{u}_{0}, w, \boldsymbol{\theta}\right),\left(\overline{\mathbf{u}}_{0}, \bar{w}, \overline{\boldsymbol{\theta}}\right)\right) \\
\quad=l\left(\overline{\mathbf{u}}_{0}, \bar{w}, \overline{\boldsymbol{\theta}}\right), \quad\left(\mathbf{u}_{0}, w, \boldsymbol{\theta}\right) \in U, \forall\left(\overline{\mathbf{u}}_{0}, \bar{w}, \overline{\boldsymbol{\theta}}\right) \in U,
\end{gathered}
$$

where $\left(^{-}\right)$expresses a variation. In addition, the bilinear forms $a(\cdot, \cdot), b(\cdot, \cdot)$ and the linear form $l(\cdot)$ are defined, respectively, in the following:

$$
\begin{aligned}
& a\left(\left(\mathbf{u}_{0}, w, \boldsymbol{\theta}\right),\left(\overline{\mathbf{u}}_{0}, \bar{w}, \overline{\boldsymbol{\theta}}\right)\right) \\
& \quad=\int_{\Omega}\left\{C_{\alpha \beta \gamma \delta}\left(u_{0 \alpha, \beta}-x_{3} \theta_{\alpha, \beta}\right)\left(\bar{u}_{0 \gamma, \delta}-x_{3} \bar{\theta}_{\gamma, \delta}\right)\right. \\
& \left.\quad+C_{\alpha \beta}^{S} \gamma_{\alpha} \bar{\gamma}_{\beta}\right\} d \Omega, \\
& b\left(\left(\mathbf{u}_{0}, w, \boldsymbol{\theta}\right),\left(\overline{\mathbf{u}}_{0}, \bar{w}, \overline{\boldsymbol{\theta}}\right)\right)=\rho \int_{\Omega}\{w \bar{w} \\
& \left.\quad+\left(u_{0 \alpha}-x_{3} \theta_{\alpha}\right)\left(\bar{u}_{0 \alpha}-x_{3} \bar{\theta}_{\alpha}\right)\right\} d \Omega, \\
& l\left(\overline{\mathbf{u}}_{0}, \bar{w}, \overline{\boldsymbol{\theta}}\right)=\int_{A}\left(f_{\alpha} \bar{u}_{0 \alpha}-m_{\alpha} \bar{\theta}_{\alpha}+q \bar{w}\right) d A \\
& \quad+\int_{\partial A_{g}}\left(N_{\alpha} \bar{u}_{0 \alpha} d s-M_{\alpha} \bar{\theta}_{\alpha}+Q \bar{w}\right) d s,
\end{aligned}
$$

where $\left\{C_{\alpha \beta \gamma \delta}\right\}_{\alpha, \beta, \gamma, \delta=1,2}$ and $\left\{C_{\alpha \beta}^{S}\right\}_{\alpha, \beta=1,2}$ denote an elastic tensor including bending and membrane components and an elastic tensor with respect to the shearing component, respectively. In addition, $\left\{\gamma_{\alpha} \equiv w_{, \alpha}-\theta_{\alpha}\right\}_{\alpha=1,2}$ expresses

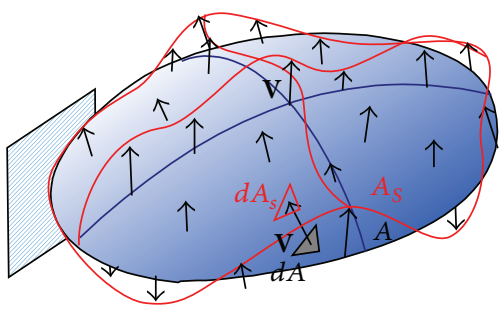

FIGURE 2: Shape variation of the thin-walled structure by $\mathbf{V}$.

the transverse shear strain tensors and the constant $\rho$ indicates material density.

Moreover, the tensor subscript notation in this paper uses Einstein's summation convention and a partial differential notation for the spatial coordinates $(\cdot)_{i}=\partial(\cdot) / \partial x_{i}$. It will be noted that $U$ in (5) is given by the following equation:

$$
\begin{aligned}
U & =\left\{\left(u_{01}, u_{02}, w, \theta_{1}, \theta_{2}\right) \in\left(H^{1}(A)\right)^{5} \mid\right. \\
& \text { satisfy the given Dirichlet condition on each subboundary }\},
\end{aligned}
$$

where $H^{1}$ is the Sobolev space of order 1.

\section{Formulation of Free-Form Optimization Problem of Thin-Walled Structure}

3.1. Domain Variation. As shown in Figure 2, consider that a linear elastic thin-walled structure having an initial domain $\Omega$, midarea $A$, boundary $\partial A$, and side surface $S$ undergoes domain variation $\mathbf{V}$ (design velocity field) in the out-ofplane direction to the midarea surface such that its domain, midarea, boundary, and side surface become $\Omega_{s}, A_{s}, \partial A_{s}$, and $S_{s}$, respectively. It is assumed that the plate thickness $h$ remains constant under the domain variation. The infinitesimal domain variation at this time can be expressed by mapping from $A$ to $A_{s}$, which is denoted by $T_{s}: X \in A \mapsto$ $X_{s}(X) \in A_{s}, 0 \leq s \leq \varepsilon$ ( $\varepsilon$ is a small integer) given by $X_{s}=T_{s}(X), A_{s}=T_{s}(A)$ [4]. The subscript $s$ expresses the iteration history of the domain variation. Assuming a shape constraint is acting on the variation in the domain, the infinitesimal variation of the domain can be expressed by

$$
T_{s+\Delta s}(X)=T_{s}(X)+\Delta s \mathbf{V},
$$

where the design velocity field $\mathbf{V}\left(X_{s}\right)=\partial T_{s}(X) / \partial s$ is given as Euler derivative of $T_{s}(\mathbf{X})$. The free-form optimization method explained later is a method for determining the optimal domain variation $\mathbf{V}$.

In order to derive the shape gradient function (i.e., sensitivity function), we use the following formula of the material derivative [15]. When the objective functional $J$ is given as a domain integral of the distributed function $\phi_{s}$,

$$
J=\int_{\Omega_{s}} \phi_{s} d \Omega
$$


The Euler derivative, or the material derivative $\dot{J}$, is given by the following expression:

$$
\dot{J}=\int_{\Omega} \phi^{\prime} d \Omega+\int_{\Gamma} \phi V_{n} d A,
$$

where $V_{n}=n_{i} V_{i}$. The vector $n$ is an outward normal unit vector to the boundary. ()$^{\prime}$ indicates a shape derivative (Lagrange derivative).

When the objective functional $J$ is given as a boundary integral of the distributed function $\phi_{s}$,

$$
J=\int_{\Gamma_{s}} \phi_{s} d A,
$$

the Euler derivative $\dot{J}$ is given by

$$
\dot{J}=\int_{\Gamma}\left\{\phi^{\prime}+\left(\phi_{, i} n_{i}+\phi H\right) V_{n}\right\} d A,
$$

where $H$ expresses twice the mean curvature of $\Gamma$ in $\mathbb{R}^{3}$.

\subsection{Minimization Problem of Squared Error of Vibration} Displacement. Let us consider a free-form optimization problem for controlling vibration displacements at a driving frequency $\omega$ on a prescribed surface or boundary to a target value. Letting the vibration equation in (5) be the constraint condition and letting the squared error of the target vibration displacements in the prescribed domain be the objective functional to be minimized, a distributed-parameter shape identification problem for finding the optimal design velocity field $\mathbf{V}$ can be formulated as shown below:

$$
\begin{aligned}
\text { Given } & A, \omega \\
\text { find } & A_{s}(\text { or } \mathbf{V}), \\
\text { that minimizes } & d\left(\mathbf{u}_{0}-\widehat{\mathbf{u}}_{0}, \mathbf{u}_{0}-\widehat{\mathbf{u}}_{0}\right) \\
& +d(w-\widehat{w}, w-\widehat{w}),
\end{aligned}
$$

subject to (5),

where $d(\cdot, \cdot)$ is the inner product defined by the following equation:

$$
d(\mathbf{u}, \mathbf{v})=\int_{A_{D}} u_{i} v_{i} d A .
$$

The notations $\widehat{\mathbf{u}}_{0}$ and $\widehat{w}$ indicate the target values per unit area of the in-plane and out-of-plane vibration displacement distributions, respectively, which are given by the prescribed domain denoted by $A_{D}$.

The Lagrange functional $L$ with respect to this problem can be expressed as

$$
\begin{aligned}
& L\left(A,\left(\mathbf{u}_{0}, w, \boldsymbol{\theta}\right),\left(\overline{\mathbf{u}}_{0}, \bar{w}, \overline{\boldsymbol{\theta}}\right)\right) \\
&= d(\mathbf{u}-\widehat{\mathbf{u}}, \mathbf{u}-\widehat{\mathbf{u}})+d(w-\widehat{w}, w-\widehat{w}) \\
&+l\left(\overline{\mathbf{u}}_{0}, \bar{w}, \overline{\boldsymbol{\theta}}\right)-a\left(\left(\mathbf{u}_{0}, w, \boldsymbol{\theta}\right),\left(\overline{\mathbf{u}}_{0}, \bar{w}, \overline{\boldsymbol{\theta}}\right)\right) \\
&+\omega^{2} b\left(\left(\mathbf{u}_{0}, w, \boldsymbol{\theta}\right),\left(\overline{\mathbf{u}}_{0}, \bar{w}, \overline{\boldsymbol{\theta}}\right)\right),
\end{aligned}
$$

where the notation with upper bar $\left(\overline{\mathbf{u}}_{0}, \bar{w}, \overline{\boldsymbol{\theta}}\right)$ which indicates variations in the state equation (see (5)) is also used as adjoint variables or Lagrange multipliers in the Lagrange functional (see (17)).

For the sake of simplicity, it is assumed that the subboundaries acted on by the nonzero external forces $\mathbf{N}, \mathbf{M}$, and $Q$ do not vary (i.e., $\mathbf{V}$ ), that the forces acting on the surfaces $\mathbf{f}, \mathbf{m}$, and $q$ do not vary with regard to the space and the iteration history $s$ (i.e., $\dot{\mathbf{f}}=\dot{\mathbf{m}}=\dot{q}=0$ ), and that the prescribed vibration displacements per unit area, $\widehat{u}_{0}$ and $\widehat{w}$, do not also vary with regard to the space and the iteration history $s$. Then, the material derivative $\dot{L}$ of the Lagrange functional can be derived as shown in (18) using the formula of material derivative [4]:

$$
\begin{aligned}
& \dot{L}= 2\left\{d\left(\mathbf{u}-\widehat{\mathbf{u}}, \mathbf{u}^{\prime}\right)+d\left(w-\widehat{w}, w^{\prime}\right)\right\}+l\left(\overline{\mathbf{u}}_{0}^{\prime}, \bar{w}^{\prime}, \overline{\boldsymbol{\theta}}^{\prime}\right) \\
&-a\left(\left(\mathbf{u}_{0}^{\prime}, w^{\prime}, \boldsymbol{\theta}^{\prime}\right),\left(\overline{\mathbf{u}}_{0}, \bar{w}, \overline{\boldsymbol{\theta}}\right)\right) \\
&+\omega^{2} b\left(\left(\mathbf{u}_{0}^{\prime}, w^{\prime}, \boldsymbol{\theta}^{\prime}\right),\left(\overline{\mathbf{u}}_{0}, \bar{w}, \overline{\boldsymbol{\theta}}\right)\right) \\
&-a\left(\left(\mathbf{u}_{0}, w, \boldsymbol{\theta}\right),\left(\overline{\mathbf{u}}_{0}^{\prime}, \bar{w}^{\prime}, \overline{\boldsymbol{\theta}}^{\prime}\right)\right) \\
&+\omega^{2} b\left(\left(\mathbf{u}_{0}, w, \boldsymbol{\theta}\right),\left(\overline{\mathbf{u}}_{0}^{\prime}, \bar{w}^{\prime}, \overline{\boldsymbol{\theta}}^{\prime}\right)\right)+\langle G \mathbf{n}, \mathbf{V}\rangle, \\
& \quad \mathbf{V} \in C_{\Theta},
\end{aligned}
$$

where

$$
\begin{aligned}
& \langle\mathrm{Gn}, \mathbf{V}\rangle \equiv \int_{A} G_{\mathrm{I}} \mathbf{n} \cdot \mathbf{V} d A+\int_{A_{D}} G_{\mathrm{II}} \mathbf{n} \cdot \mathbf{V} d A \\
& =\int_{A}\left(G_{\mathrm{I}}+G_{\mathrm{II}}\right) \mathbf{n} \cdot \mathbf{V} d A, \\
& G_{\mathrm{I}}=-\left\{C_{\alpha \beta \gamma \delta}\left(u_{0 \alpha, \beta}+\frac{h}{2} \theta_{\alpha, \beta}\right)\left(\bar{u}_{0 \gamma, \delta}+\frac{h}{2} \bar{\theta}_{\gamma, \delta}\right)\right. \\
& \left.-C_{\alpha \beta \gamma \delta}\left(u_{0 \alpha, \beta}-\frac{h}{2} \theta_{\alpha, \beta}\right)\left(\bar{u}_{0 \gamma, \delta}-\frac{h}{2} \bar{\theta}_{\gamma, \delta}\right)\right\} \\
& +\left[\rho \omega^{2}\left\{w \bar{w}+\left(u_{0 \alpha}+\frac{h}{2} \theta_{\alpha}\right)\left(\bar{u}_{0 \alpha}+\frac{h}{2} \bar{\theta}_{\alpha}\right)\right\}\right. \\
& \left.\quad-\rho \omega^{2}\left\{w \bar{w}+\left(u_{0 \alpha}-\frac{h}{2} \theta_{\alpha}\right)\left(\bar{u}_{0 \alpha}-\frac{h}{2} \bar{\theta}_{\alpha}\right)\right\}\right] \\
& +H f_{\alpha} \bar{u}_{0 \alpha}-H m_{\alpha} \bar{\theta}_{\alpha}+H q \bar{w}, \\
& G_{\mathrm{II}}=H\left\{\left(u_{01}-\widehat{u}_{01}\right)^{2}+\left(u_{02}-\widehat{u}_{02}\right)^{2}+(w-\widehat{w})^{2}\right\},
\end{aligned}
$$

where the notation $\mathbf{n}$ indicates an outward unit normal vector on the surface $A$. The relationship $(\mathbf{n} \cdot \mathbf{V}) \mathbf{n}=\left(\mathbf{n}^{\text {top }}\right.$. $\mathbf{V}) \mathbf{n}^{\text {top }}=-\left(\mathbf{n}^{\text {btm }} \cdot \mathbf{V}\right) \mathbf{n}^{\text {btm }}$ for the out-of-plane shape variation is assumed to derive (19) by using the notations $\mathbf{n}^{\text {top }}$ and $\mathbf{n}^{\text {btm }}$, outward unit normal vectors on the top and bottom surface, respectively. Moreover, the notation $G \mathbf{n}$ denotes the shape gradient function, and $G$ is called the shape gradient density function. Equations (20) and (21) express 

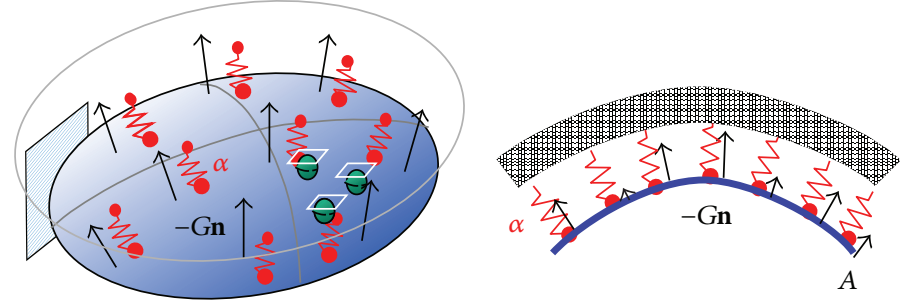

FIGURE 3: $H^{1}$ gradient method for thin-walled structure under Robin boundary condition.

the sensitivity functions (i.e., shape gradient functions) for this problem. Additionally, the notation $H$ denotes twice the mean curvature of the surface and $C_{\Theta}$ expresses the admissible function space that satisfies the constraints of shape variation. The notations $(\cdot)^{\prime}$ and $(\cdot)$ are the shape derivative and the material derivative with respect to the domain variation, respectively [16].

The optimality conditions of the Lagrange functional $L$ with respect to the state variable $\left(\mathbf{u}_{0}, w, \boldsymbol{\theta}\right)$ and the adjoint variable $\left(\overline{\mathbf{u}}_{0}, \bar{w}, \overline{\boldsymbol{\theta}}\right)$ are expressed as

$$
\begin{aligned}
& a\left(\left(\mathbf{u}_{0}, w, \boldsymbol{\theta}\right),\left(\overline{\mathbf{u}}_{0}^{\prime}, \bar{w}^{\prime}, \overline{\boldsymbol{\theta}}^{\prime}\right)\right) \\
& -\omega^{2} b\left(\left(\mathbf{u}_{0}, w, \boldsymbol{\theta}\right),\left(\overline{\mathbf{u}}_{0}^{\prime}, \bar{w}^{\prime}, \overline{\boldsymbol{\theta}}^{\prime}\right)\right)=l\left(\overline{\mathbf{u}}_{0}^{\prime}, \bar{w}^{\prime}, \overline{\boldsymbol{\theta}}^{\prime}\right), \\
& \forall\left(\mathbf{u}_{0}, w, \boldsymbol{\theta}\right) \in U, \forall\left(\overline{\mathbf{u}}_{0}^{\prime}, \bar{w}^{\prime}, \overline{\boldsymbol{\theta}}^{\prime}\right) \in U, \\
& a\left(\left(\mathbf{u}_{0}^{\prime}, w^{\prime}, \boldsymbol{\theta}^{\prime}\right),\left(\overline{\mathbf{u}_{0}}, \bar{w}, \overline{\boldsymbol{\theta}}\right)\right) \\
& -\omega^{2} b\left(\left(\mathbf{u}_{0}^{\prime}, w^{\prime}, \boldsymbol{\theta}^{\prime}\right),\left(\overline{\mathbf{u}}_{0}, \bar{w}, \overline{\boldsymbol{\theta}}\right)\right) \\
& =2\left\{d\left(\mathbf{u}-\widehat{\mathbf{u}}, \mathbf{u}^{\prime}\right)+d\left(w-\widehat{w}, w^{\prime}\right)\right\}, \\
& \forall\left(\overline{\mathbf{u}}_{0}, \bar{w}, \overline{\boldsymbol{\theta}}\right) \in U, \forall\left(\mathbf{u}_{0}^{\prime}, w^{\prime}, \boldsymbol{\theta}^{\prime}\right) \in U .
\end{aligned}
$$

When the optimality conditions are satisfied, (18) becomes

$$
\dot{L}=\langle G \mathbf{n}, \mathbf{V}\rangle, \quad \mathbf{V} \in C_{\Theta} .
$$

Equation (22) is the governing equation of the frequency response analysis for determining the state variable $\left(\mathbf{u}_{0}, w, \boldsymbol{\theta}\right)$ that coincides with (5), and (23) is the governing equation of the adjoint frequency analysis for determining the adjoint variable $\left(\overline{\mathbf{u}}_{0}, \bar{w}, \overline{\boldsymbol{\theta}}\right)$. The shape gradient functions $G$ and $G_{D}$ are calculated using the results obtained for $\left(\mathbf{u}_{0}, w, \boldsymbol{\theta}\right)$ and $\left(\overline{\mathbf{u}}_{0}, \bar{w}, \overline{\boldsymbol{\theta}}\right)$.

Then, the shape gradient function derived is applied to the free-form optimization method for thin-walled structures to determine the optimal velocity field or optimal shape.

\section{Free-Form Optimization Method for Thin-Walled Structures}

The free-form optimization method described here is based on the $H^{1}$ gradient method, which is also called the traction method and is a type of gradient method in a Hilbert space. It is a node-based optimization method that can treat all nodes as design variables without requiring any design variable parameterization. This capability makes it possible to obtain the optimal free form of thin-walled structures. The original traction method was proposed by Azegami in 1994 [11]. The authors have been modifying the original method for shell optimization [10].

As illustrated in Figure 3, with this method, the negative shape gradient function $G$ is applied as a distributed surface force to a pseudoelastic thin-walled structure in the normal direction to the surface under a Robin boundary condition, that is, an elastic support condition (spring constant $\alpha>0$ ). The shape gradient function is not used directly to vary the shape but instead is replaced by a force. The stiffness tensor, which is used as a positive definite tensor which is needed for a gradient method in a Hilbert space, serves as a mapping function and as a smoother (or a filter for jagged shape problem) for maintaining mesh smoothness. This approach, which can be regarded as one type of filtering approach, makes it possible both to reduce the objective functional and to maintain surface smoothness, that is, mesh regularity, which is the most distinctive feature of the proposed method.

We call the fictitious elastic analysis to determine the optimal shape variation or the design velocity $\mathbf{V}$ "velocity analysis." The original shape is updated to a new shape by adding the obtained optimal shape variation $\mathbf{V}$. In the velocity analysis, constraint conditions can be set arbitrarily by designers according to the requirements in various practical design problems.

Considering the design velocity $\mathbf{V}=\left\{V_{i}\right\}_{i=1,2,3}$ as a combination of the in-plane velocity $\left\{V_{0_{\beta}}\right\}_{\beta=1,2}$ and the outof-plane velocity $V_{3}$, the governing equation of the velocity analysis for $\mathbf{V}=\left(V_{0_{1}}, V_{0_{2}}, V_{3}\right)$ is expressed as (25) with the definition of $C_{\Theta}$ in (26). Consider

$$
\begin{aligned}
& a\left(\left(V_{0_{1}}, V_{0_{2}}, V_{3}, \boldsymbol{\theta}\right),\left(\overline{\mathbf{u}}_{0}, \bar{w}, \overline{\boldsymbol{\theta}}\right)\right) \\
& +\alpha\left\langle(\mathbf{V} \cdot \mathbf{n}) \mathbf{n},\left(\overline{\mathbf{u}}_{0}, \bar{w}, \overline{\boldsymbol{\theta}}\right)\right\rangle \\
& =-\left\langle G_{A} \mathbf{n},\left(\overline{\mathbf{u}}_{0}, \bar{w}, \overline{\boldsymbol{\theta}}\right)\right\rangle, \\
& \quad\left(V_{0_{1}}, V_{0_{2}}, V_{3}, \boldsymbol{\theta}\right) \in C_{\Theta}, \forall\left(\overline{\mathbf{u}}_{0}, \bar{w}, \overline{\boldsymbol{\theta}}\right) \in C_{\Theta},
\end{aligned}
$$


where $C_{\Theta}$ is expressed as

$$
C_{\Theta}=\left\{\left(V_{0_{1}}, V_{0_{2}}, V_{3}, \theta_{1}, \theta_{2}\right) \in\left(H^{1}(A)\right)^{5} \mid\right.
$$

satisfy Robin condition for shape variation on $S\}$.

Here, we can confirm that the domain variation $\mathbf{V}$ determined by the velocity analysis reduces the Lagrange functional $L$. When the eigenvalue equation, the adjoint equation, and the constraints are satisfied, the perturbation expansion of the Lagrange functional $L$ can be written as

$$
\Delta L=\langle G \mathbf{n}, \Delta s(\mathbf{V}, \boldsymbol{\theta})\rangle+O\left(|\Delta s|^{2}\right) .
$$

Considering the arbitrariness of $\left(\overline{\mathbf{u}}_{0}, \bar{w}, \overline{\boldsymbol{\theta}}\right)$ in (25), (25) is substituted into (27) to obtain

$$
\begin{aligned}
& \Delta L \\
& \simeq\langle G \mathbf{n}, \Delta s(\mathbf{V}, \boldsymbol{\theta})\rangle \\
& \quad-\{a((\mathbf{V}, \boldsymbol{\theta}), \Delta s(\mathbf{V}, \boldsymbol{\theta}))+\alpha\langle(\mathbf{V} \cdot \mathbf{n}) \mathbf{n}, \Delta s(\mathbf{V}, \boldsymbol{\theta})\rangle\} .
\end{aligned}
$$
(25),

Taking into account the bilinear form on the left side of

$$
a((\mathbf{V}, \boldsymbol{\theta}),(\bar{w}, \overline{\boldsymbol{\theta}}))+\alpha\langle(\mathbf{V} \cdot \mathbf{n}) \mathbf{n},(\mathbf{V}, \boldsymbol{\theta})\rangle>0,
$$

which is based on $\alpha>0, \Delta s>0$, and the positive definitiveness of the bilinear form

$$
\begin{aligned}
& \exists \beta>0: a((\mathbf{V}, \boldsymbol{\theta}),(\mathbf{V}, \boldsymbol{\theta})) \geq \beta\|(\mathbf{V}, \boldsymbol{\theta})\|^{2}, \\
& (\mathbf{V}, \boldsymbol{\theta}) \in C_{\Theta},
\end{aligned}
$$

the following relationship is obtained:

$$
\Delta L<0 .
$$

In problems where convexity is assured, this relationship definitely reduces the Lagrange functional in the process of changing the domain using the design velocity field $\mathbf{V}$ determined by (25). The stiffness tensor in the governing equation (see (25)) serves as a smoother for maintaining mesh regularity.

As shown in Figure 4, the optimal free-form thin-walled structure is obtained by repeating a process consisting of (1) a frequency response analysis and an adjoint analysis, (2) a sensitivity analysis for calculating the shape gradient function, (3) a velocity analysis, and (4) shape updating. The analyses in (1) and (3) are conducted using an ordinary general-purpose FEM code. The calculations in (2) and (4) can be performed easily using $\mathrm{C}$ language.

\section{Results of Numerical Analysis}

The proposed method was applied to two fundamental design problems in order to confirm its validity and practical utility for thin-walled structures. In each design problem, all plate thickness was $h=1$, Young's modulus was 200000, and the density was $\rho=7.9 \times 10^{-9}$. The frequency response analysis

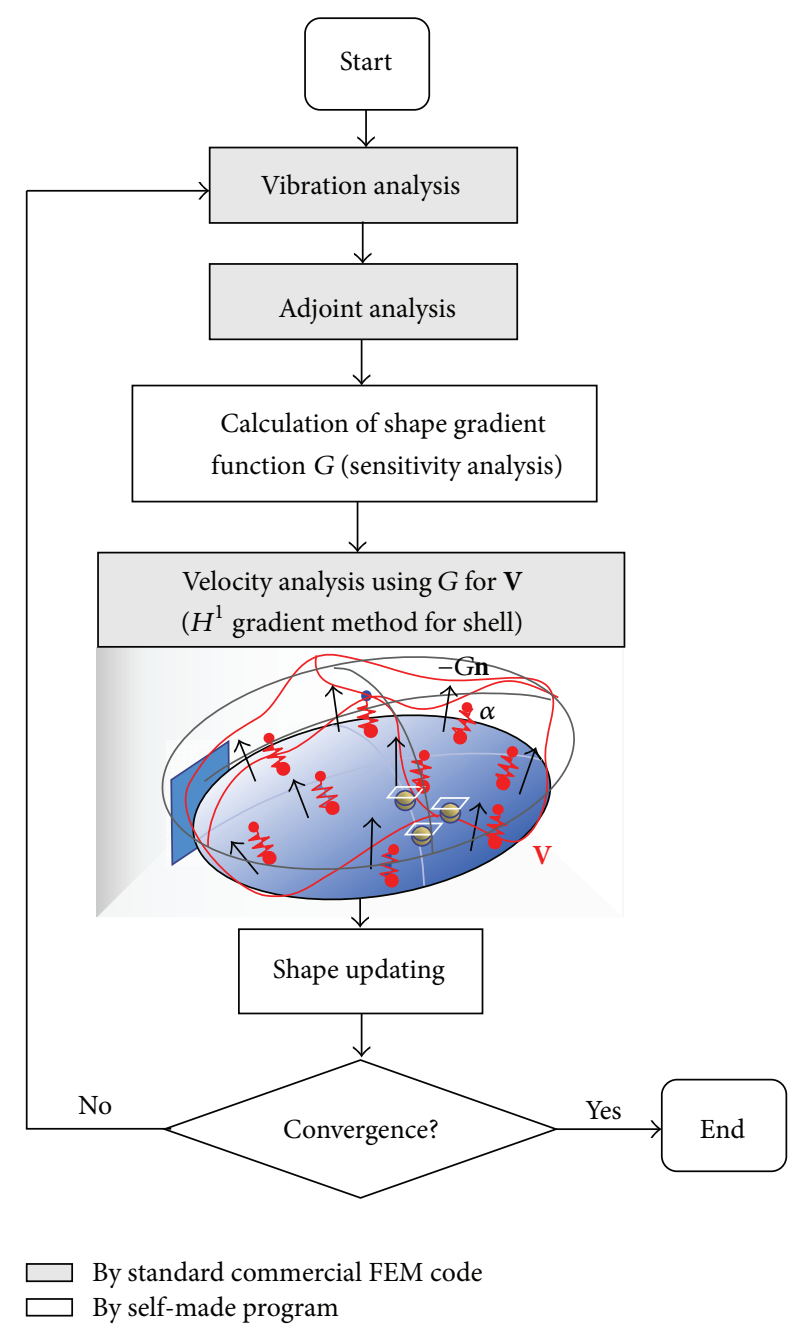

FIGURE 4: Schematic of the parameter-free optimization method.

was performed by the MSC NASTRAN finite element code, in which linear triangular shell elements (CTRIA3 elements) were employed to create more detailed and smooth surface shapes of shells. Moreover, in the velocity analysis, the earth spring constant was set at $\alpha=2 \times 10^{4}$, which was 1.2 times the bending rigidity. It was discussed in our previous work $[10,12]$ that $\alpha=2 \times 10^{4}$ is more suitable for designing clear beads around the initial surface.

5.1. Half-Cylindrical Shell Model. Figure 7 shows the initial shape and boundary conditions of a half-cylindrical shell, which is modelled by 1092 nodes and 2088 elements. In the frequency response analysis, both the top and bottom edges were simply supported and a center line was uniformly excited in the vertical direction by applying a frequency of $1350 \mathrm{~Hz}$, which is near the first natural frequency $(1375.68 \mathrm{~Hz})$. The resultant vibration mode is shown in Figure 5(c). The excited boundary was defined as the amplitudes prescribed boundary. The target amplitude distribution was defined as 1/10 (uniform distribution) of the maximum amplitude in the excited direction at the amplitude prescribed 


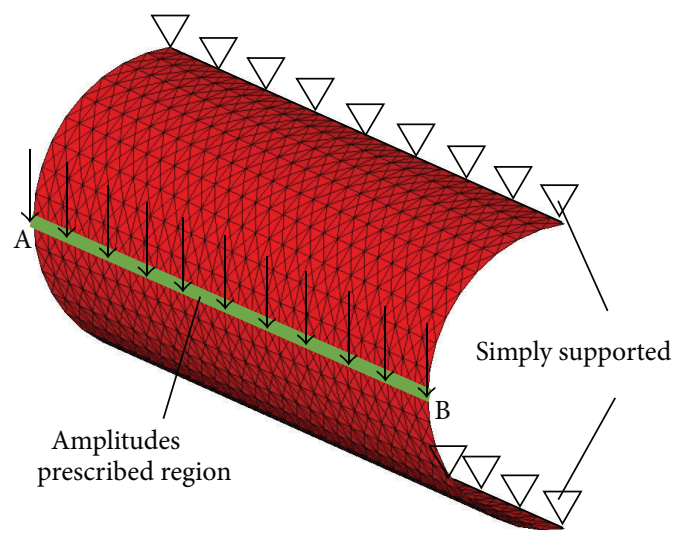

(a) Frequency response analysis

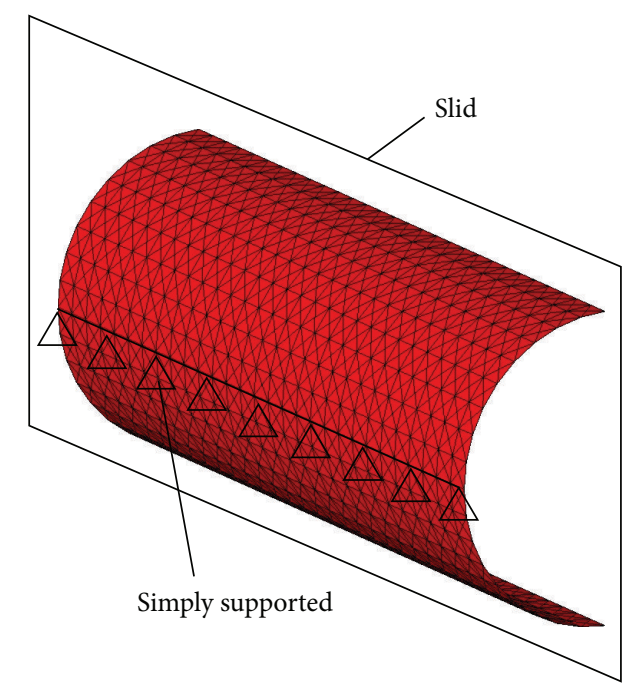

(b) Velocity analysis

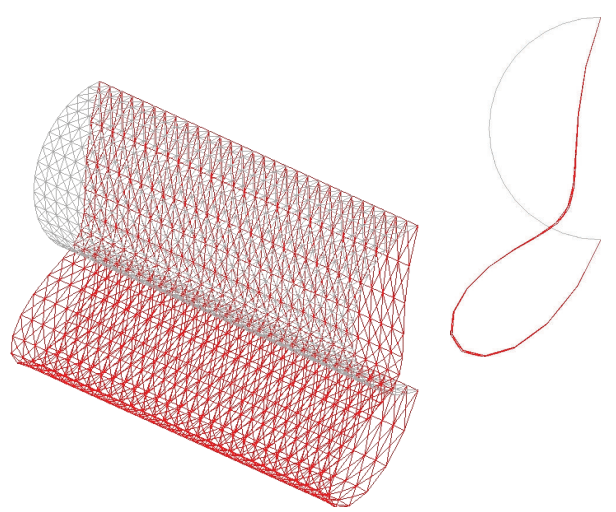

(c) Vibration mode of initial shape at $1350 \mathrm{~Hz}$

FIGURE 5: Initial shape and boundary conditions of half-cylindrical shell model $(1350 \mathrm{~Hz})$.

boundary of the initial shape, as shown in Figure 5(a). The constraint conditions of the velocity analysis can be freely defined according to the practical shape design requirements but must be in accordance with the assumption in the derivation of the shape gradient functions as mentioned in Section 3.2. In the velocity analysis of this design problem, the top and bottom edges were subjected to sliding restraints, as shown in Figure 5(b), and the excited location of the frequency response was simply supported. The optimal shape obtained and the iteration convergence histories are shown in Figures 6(a) and 6(b), respectively. The results in Figure 6(a) show the formation of shallow beads along the free ends on the right and left sides. It is seen in Figure 6(b) that the objective functional converged almost to zero. There was virtually no change in the volume. Figure $6(\mathrm{c})$ compares the amplitude distributions at the amplitude prescribed location, that is, section A-B in Figure 5(a). The graph shows that the amplitude distribution of the obtained shape agreed well with the target distribution. The frequency response curves of the initial shape and the obtained shape are compared in Figure 6(d). The calculated results indicate that the primary natural frequency was shifted upward by approximately
$200 \mathrm{~Hz}$ and that the amplitude of the obtained shape was 1/10 of that of the initial shape as intended.

Using the same model and boundary conditions as in Figure 5, calculations were performed when the target prescribed amplitude value was defined as $1 / 100$ and $1 / 1000$ of the maximum amplitude in the excited direction at the amplitude prescribed boundary of the initial shape. The purpose here was to investigate the influence of the prescribed value on the optimal shape. The shapes obtained for a prescribed value of 1/100 of the maximum amplitude and frequency response curve are shown in Figures 7(a) and 7(b), respectively. The corresponding results that were obtained for a prescribed value of 1/1000 are shown in Figures 8(a) and 8(b). Both sets of results indicate that bead formation shifted the first natural frequency upward substantially. As the prescribed amplitude was reduced, the number of beads increased and deeper beads were formed, which resulted in higher stiffness.

5.2. Table-Shaped Model. Figure 9 shows the initial shape and boundary conditions of a table-like model with 3219 nodes and 6240 elements. In the frequency response analysis 


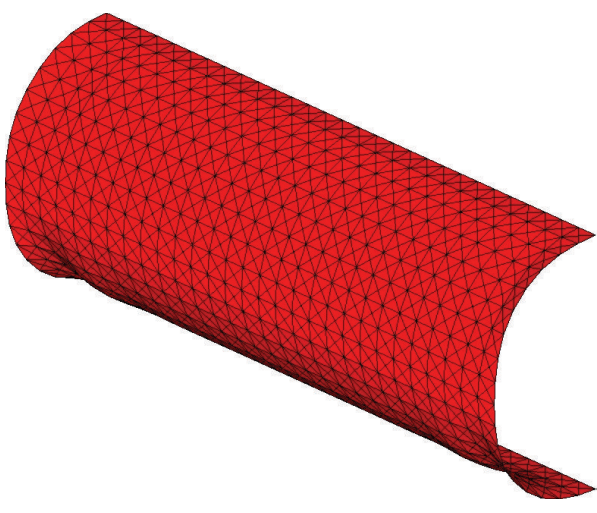

(a) Obtained shape

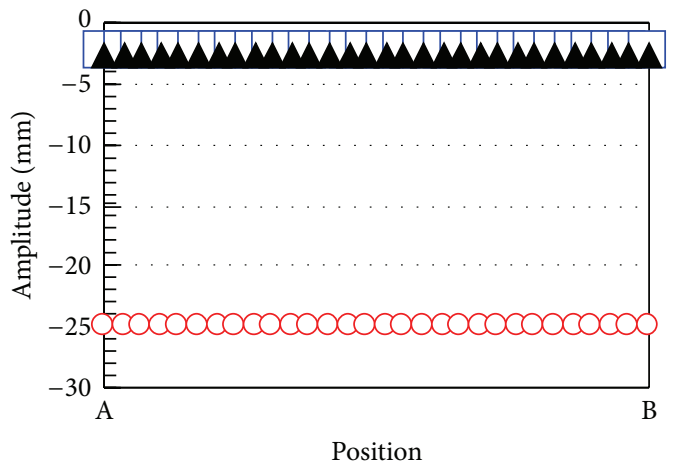

$-\square-$ Desired

- Optimal

- - Initial

(c) Amplitudes at the prescribed boundary

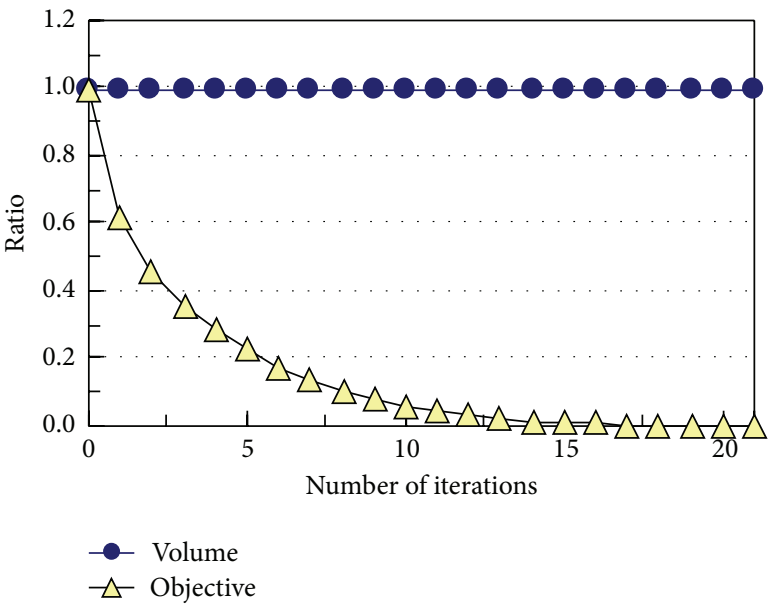

(b) Iteration histories

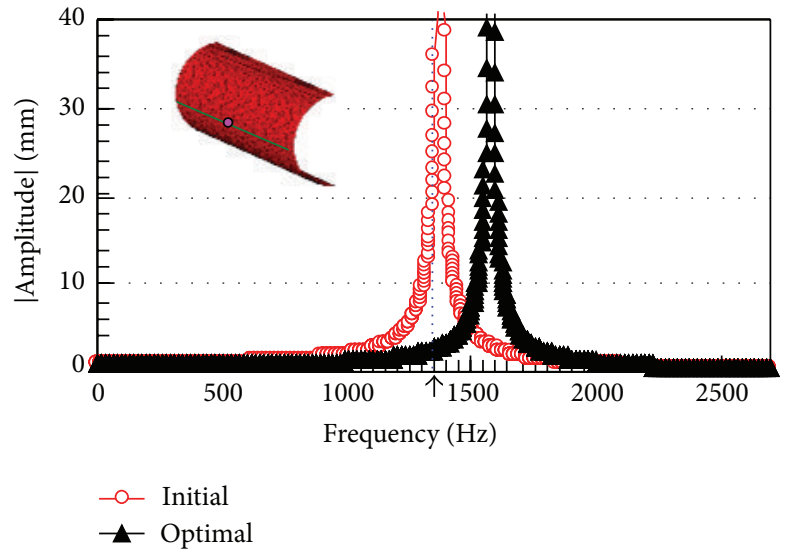

(d) Frequency response curves

FIGURE 6: Optimization results of half-cylindrical shell model at $1350 \mathrm{~Hz}$.

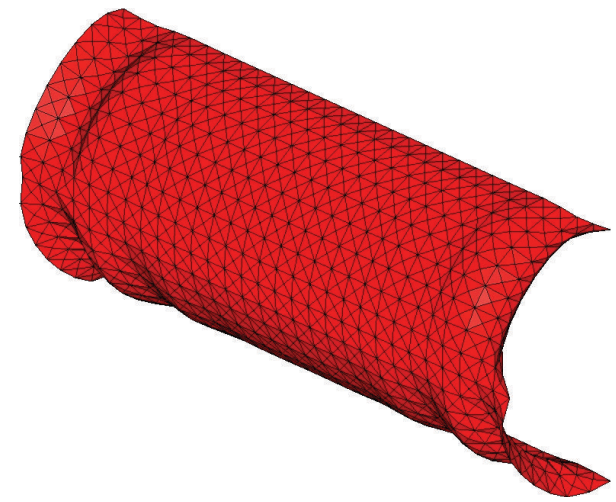

(a) Obtained shape



(b) Comparison of frequency response curves of cylindrical shell

FIGURE 7: Optimization results for $u=0.01 \max \left(u^{\text {init }}\right)$ of half-cylindrical shell. 


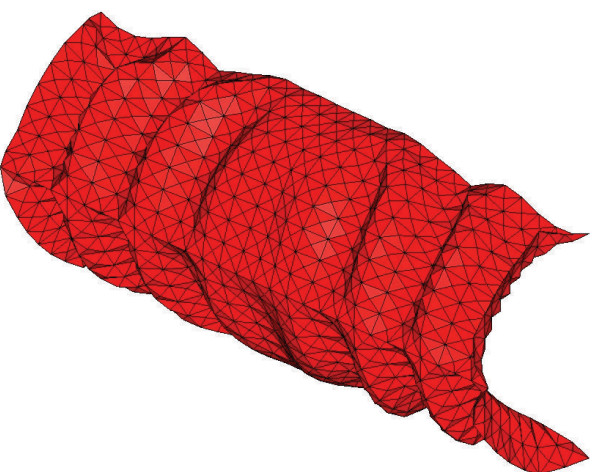

(a) Obtained shape

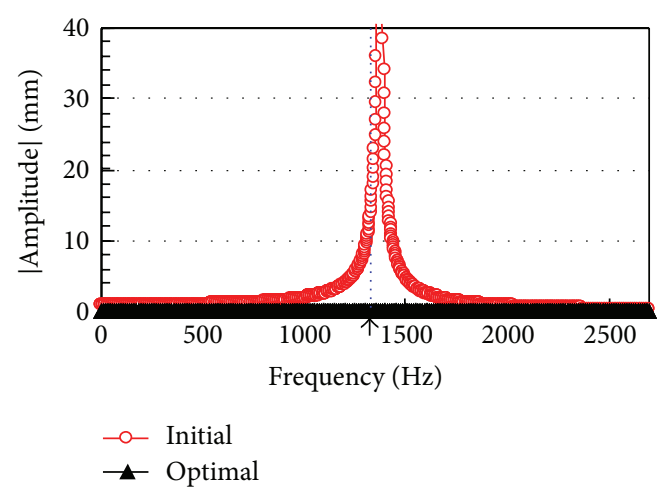

(b) Comparison of frequency response curves of cylindrical shell

FIGURE 8: Optimization results for $u=0.001 \max \left(u^{\text {init }}\right)$ of half-cylindrical shell.

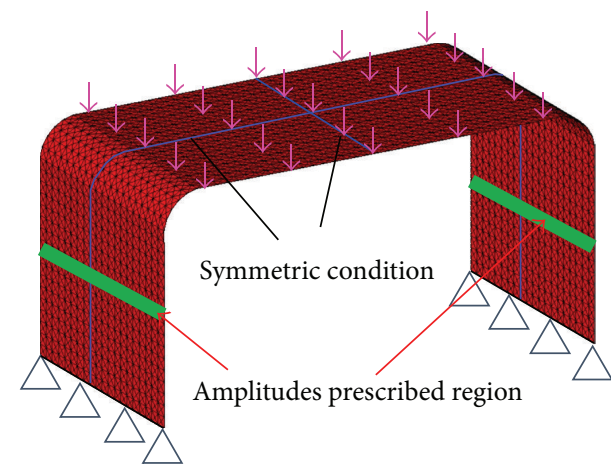

Simply supported

(a) Frequency response analysis

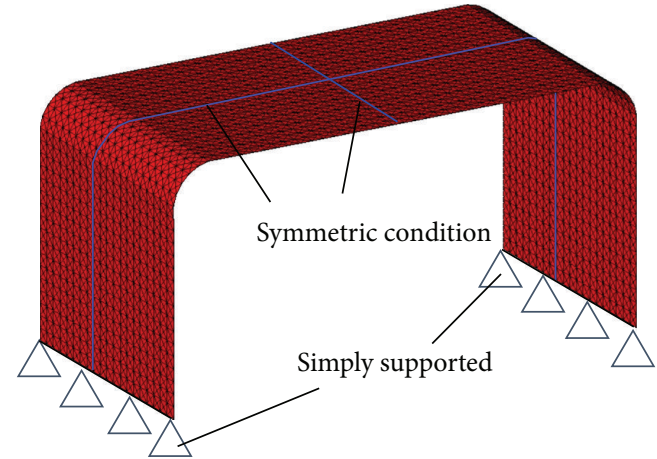

(b) Velocity analysis

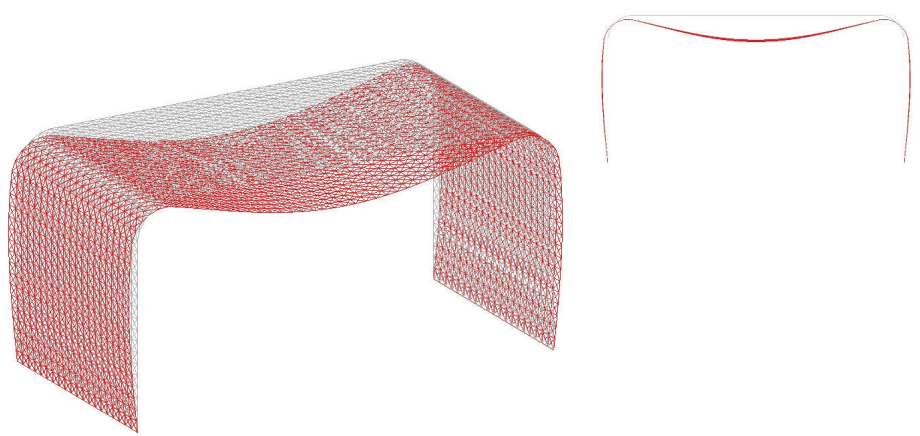

(c) Vibration mode of initial shape at $350 \mathrm{~Hz}$

FIGURE 9: Initial shape and boundary conditions of table-shaped model $(350 \mathrm{~Hz})$.

(Figure 9(a)), the bottom edges were simply supported and symmetric conditions were defined for the center lines of the top and side surfaces. The top surface was uniformly excited by applying a frequency of $350 \mathrm{~Hz}$, which was close to the first natural frequency $(382.96 \mathrm{~Hz})$. The resultant vibration mode is shown in Figure 9(c). As shown in Figure 9(a), the prescribed boundary of the amplitudes was defined as a line approximately in the center of both sides, and the target amplitude distribution was defined as 1/1000 (uniform distribution) of the maximum amplitude in the excited direction at the amplitude prescribed boundary of the initial shape. In the velocity analysis, both edges at the bottom were simply supported as shown in Figure 9(b), and symmetric conditions were defined in the same way as in the frequency response analysis. The optimal shape obtained and the iteration convergence histories are shown in Figures 10(a) and 

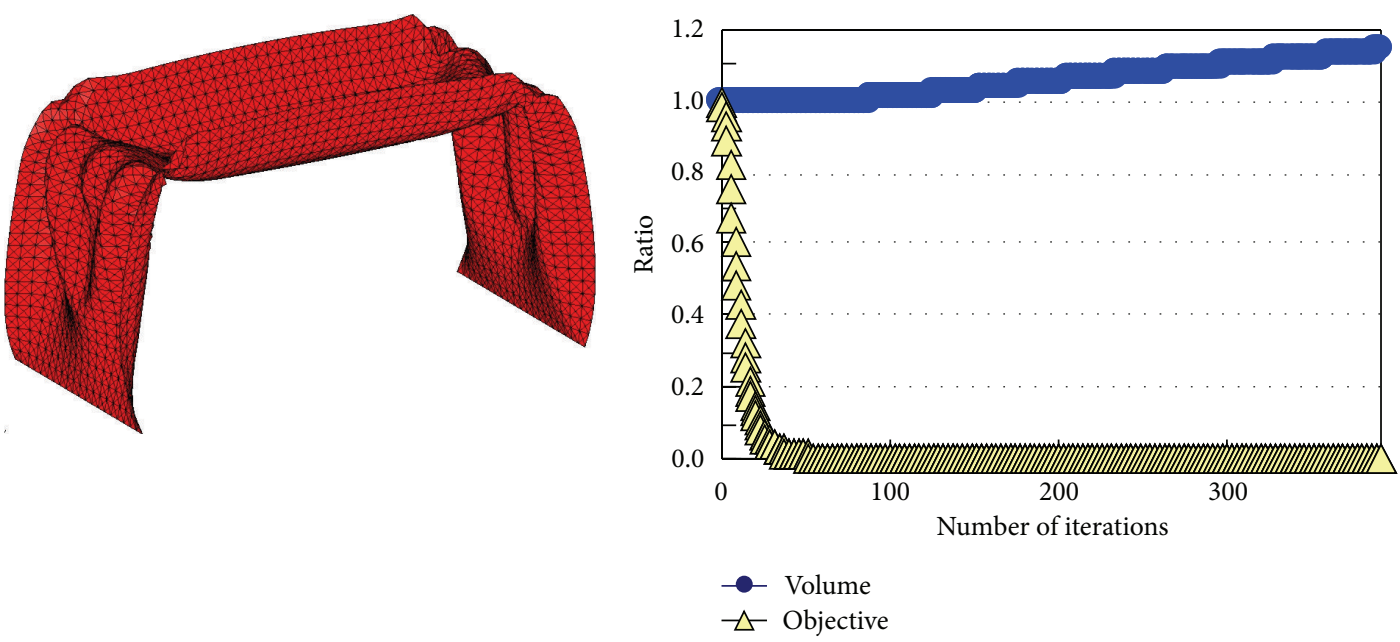

(a) Obtained shape

(b) Iteration histories

FIGURE 10: Optimization results of table-shaped model $(350 \mathrm{~Hz})$.

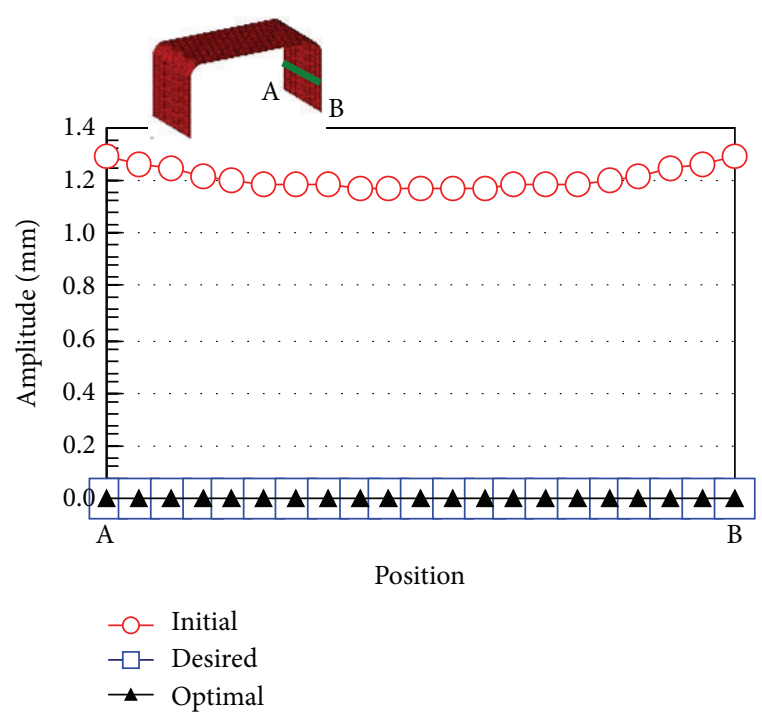

(a) Amplitudes at the prescribed boundary

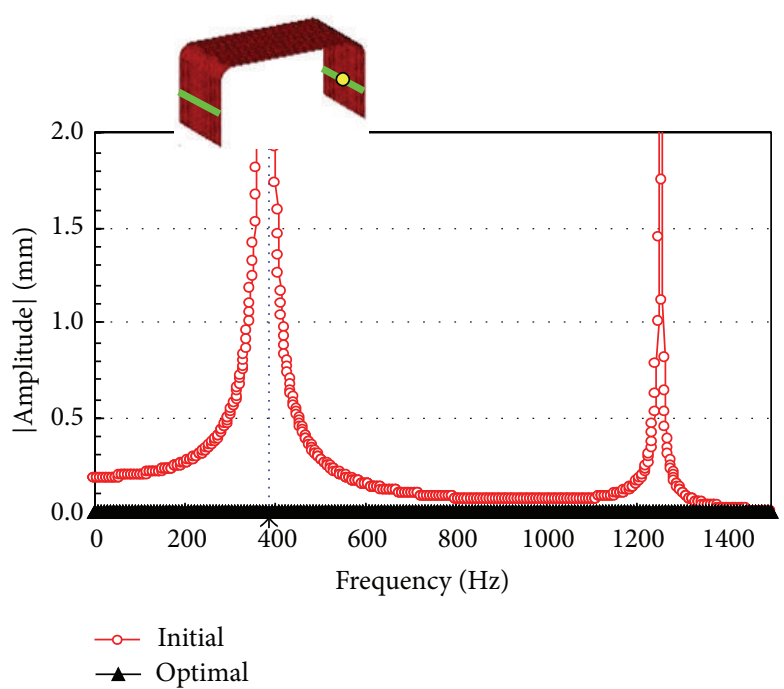

(b) Frequency response curves

FIGURE 11: Comparison of amplitude distributions and frequency responses (table-shaped model).

10(b), respectively. The optimal shape in Figure 10(a) shows the formation of deep beads for increasing the stiffness of the sides and top surface. It is observed in Figure 10(b) that the objective functional converged almost to zero. The calculated results indicate that the volume increased by approximately $18 \%$.

Figure 11(a) compares the amplitude distributions at the amplitude prescribed location, that is, section $A-B$ in Figure 9(a). In this example as well, the amplitude distribution of the obtained shape agreed well with the target distribution. The frequency response curves of the initial shape and the obtained shape are compared in Figure 11(b). These frequency response curves are for the center point of section A-B in Figure 9(a). The calculated results indicate that the 1st natural frequency was shifted upward over $1000 \mathrm{~Hz}$ and that the amplitude at the excitation frequency was substantially reduced to the prescribed value from that of the initial shape as intended.

\section{Conclusions}

This paper has described the free-form optimization method for controlling a vibration mode in a frequency response problem of thin-walled structures. A shape identification problem, in which the squared error of the desired vibration displacement distribution was defined as the objective functional, was formulated as a distributed-parameter shape 
optimization problem. The shape gradient function with respect to out-of-plane shape variation was derived theoretically and applied to the developing free-form optimization method. With this approach, the objective functional converged almost to zero in all the sample design problems considered, and beaded shapes coinciding with the prescribed amplitude distribution were obtained as intended, thereby validating the practical utility of the proposed method. The use of this method makes it possible to find the optimal shape that coincides with the desired amplitude distribution in frequency response problems of thin-walled structures. The results presented also confirmed that setting a small prescribed value in relation to the initial shape results in the formation of beads for increasing the stiffness, which has the effect of shifting the natural frequency upward substantially.

\section{Conflict of Interests}

The authors declare that there is no conflict of interests regarding the publication of this paper.

\section{Acknowledgment}

This work was supported in part by the Yazaki Memorial Foundation for Science and Technology.

\section{References}

[1] E. Ramm, K.-U. Bletzinger, and R. Reitinger, "Shape optimization of shell structures," Journal of the International Association for Shell and Spatial Structures, vol. 34, no. 112, pp. 103-121, 1993.

[2] N. V. R. Rao and E. Hinton, "Analysis and optimization of prismatic plate and shell structures with curved planform-II. Shape optimization," Computers \& Structures, vol. 52, no. 2, pp. 341-351, 1994.

[3] T. Lindby and J. L. T. Santos, "Shape optimization of threedimensional shell structures with the shape parametrization of a CAD system," Structural Optimization, vol. 18, no. 2-3, pp. 126133, 1999.

[4] K. K. Choi and N. H. Kim, Structural Sensitivity Analysis and Optimization 1, Springer, New York, NY, USA, 2005.

[5] V. Braibant and C. Fleury, "Shape optimal design using bsplines," Computer Methods in Applied Mechanics and Engineering, vol. 44, no. 3, pp. 247-267, 1984.

[6] K.-U. Bletzinger, M. Firl, J. Linhard, and R. Wüchner, "Optimal shapes of mechanically motivated surfaces," Computer Methods in Applied Mechanics and Engineering, vol. 199, no. 5-8, pp. 324333, 2010.

[7] M. Firl and K.-U. Bletzinger, "Shape optimization of thin walled structures governed by geometrically nonlinear mechanics," Computer Methods in Applied Mechanics and Engineering, vol. 237-240, pp. 107-117, 2012.

[8] M. Firl, R. Wuchner, and K.-U. Bletzinger, "Regularization of shape optimization problems using FE-based parametrization," Structural and Multidisciplinary Optimization, vol. 47, no. 4, pp. 507-521, 2013.

[9] K.-U. Bletzinger, "A consistent frame for sensitivity filtering and the vertex assigned morphing of optimal shape," Structural and Multidisciplinary Optimization, vol. 49, no. 6, pp. 873-895, 2014.
[10] M. Shimoda and Y. Liu, "A non-parametric free-form optimization method for shell structures," Structural and Multidisciplinary Optimization, vol. 50, no. 3, pp. 409-423, 2014.

[11] H. Azegami, "A solution to domain optimization problems," Transactions of the Japan Society of Mechanical EngineersSeries A, vol. 60, no. 574, pp. 1479-1486, 1994 (Japanese).

[12] Y. Liu and M. Shimoda, "A non-parametric solution to shape identification problem of free-form shells for desired deformation mode," Computers \& Structures, vol. 144, pp. 1-11, 2014.

[13] M. Shimoda, "Free-form optimization method for designing automotive shell structures," SAE International Journal of Passenger Cars-Electronic and Electrical Systems, vol. 4, pp. 42-54, 2011.

[14] E. Reissner, "The effect of transverse shear deformation on the bending of elastic plates," Journal of Applied Mechanics, vol. 67, pp. A-69-A-77, 1945.

[15] K. K. Choi and N. H. Kim, Structural Sensitivity Analysis and Optimization 2, Springer, New York, NY, USA, 2005.

[16] J. Sokolowski and J. Zolesio, Introduction to Shape Optimization, Shape Sensitivity Analysis, Springer, New York, NY, USA, 1991. 

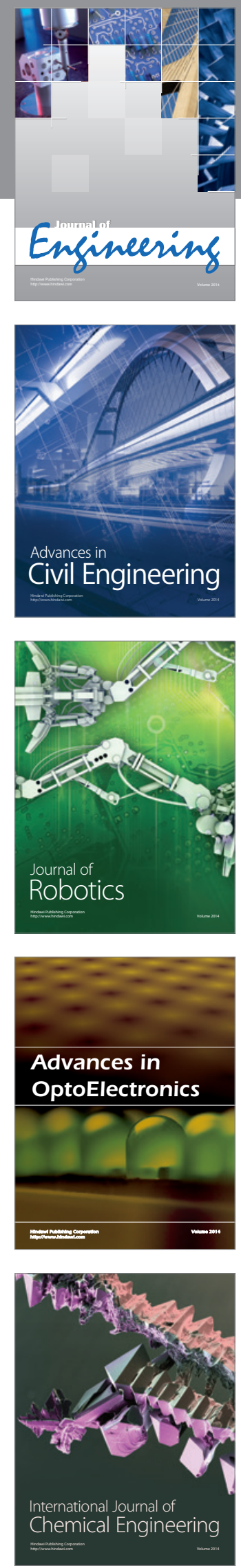

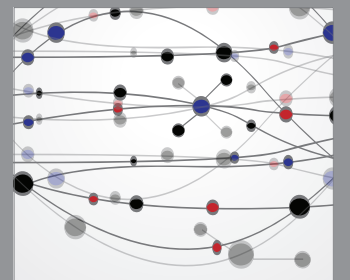

The Scientific World Journal
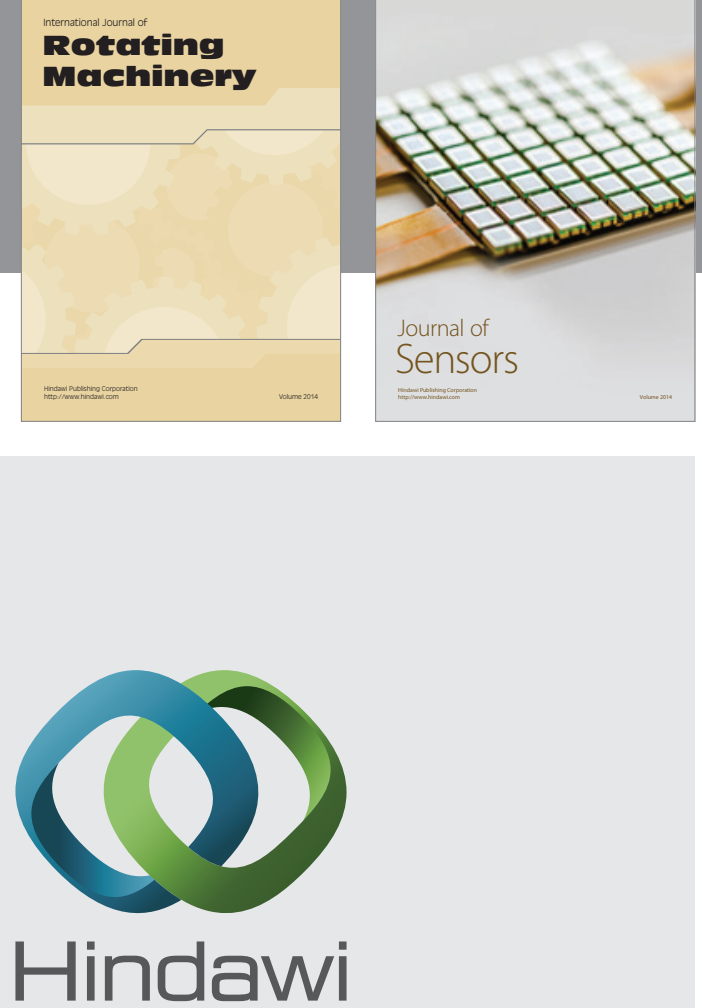

Submit your manuscripts at http://www.hindawi.com
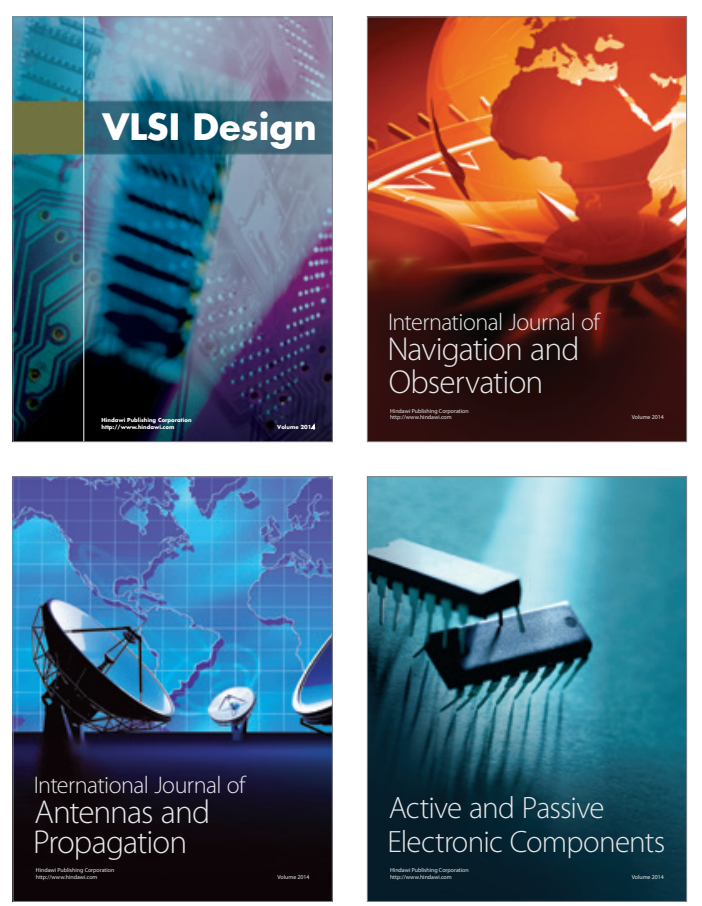
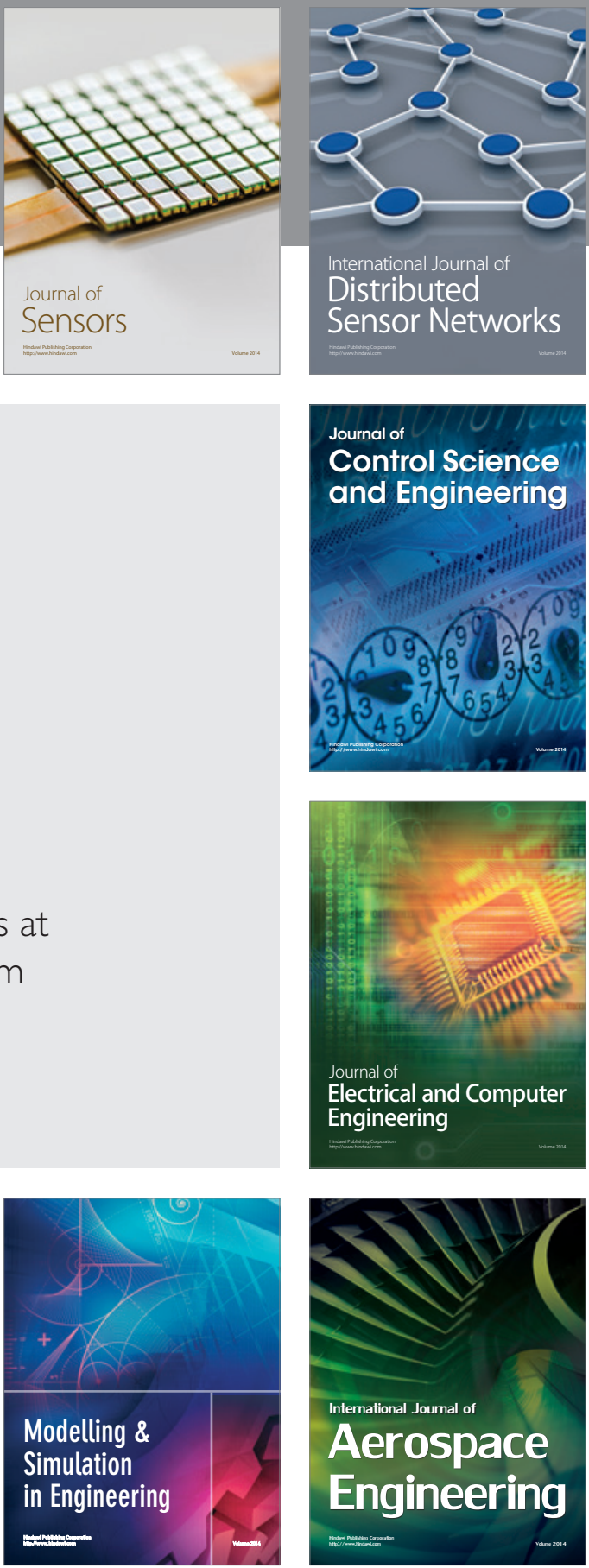

Journal of

Control Science

and Engineering
